\title{
INTELIGENCIA EMOCIONAL: RETRATO E IMPORTANCIA EN PROFESIONALES DE LA SALUD (TRABAJADORES DEL SECTOR FITNESS, CIRUJANOS Y PODÓLOGOS)
}

\section{EMOTIONAL INTELLIGENCE: PORTRAIT AND SIGNIFICANCE IN HEALTHCARE WORKERS (FITNESS CENTER WORKERS, SURGEONS AND PODIATRIST)}

Javier Simón Grima. Universidad de Zaragoza. España.

javisimongrima@gmail.com

\section{RESUMEN}

La inteligencia emocional (IE) se refiere a la forma en que las personas perciben, valoran y expresan emociones, y a las habilidades personales, cognitivas y/o sociales que determinan la eficacia de las personas para comprender y afrontar los problemas diarios. Hasta hace unas décadas, poco se sabía acerca de la IE ya que, se consideraba a la inteligencia cognitiva como el único predictor de "éxito" en una persona. No obstante, en los últimos años, ha habido un creciente interés de los investigadores por identificar las connotaciones que tiene la IE con la salud. En esta revisión se muestran los resultados de diferentes investigaciones sobre IE y variables relacionadas con la salud y el desempeño laboral en poblaciones poco estudiadas relacionadas con la salud (trabajadores del sector fitness, cirujanos y podólogos). Se proponen algunas claves para mejorar la IE con el objetivo de reducir el estrés, mejorar la salud mental y social y aumentar la eficacia del afrontamiento ante diferentes agentes estresores (carga laboral, enfermedad o muerte de un familiar, etc.).

PALABRAS CLAVE: Educación, emoción, estrés, inteligencia emocional, salud

\section{ABSTRACT}

The emotional intelligence (EI) refers to how people perceive, value and show emotions, as well as those individual, cognitive and/or social skills which define the efficacy of the people to understanding and confronting the daily problems. Up until a few decades ago, there wasn't much knowledge about El. Nonetheless, recently, there has recently been an increasing attention by research community for identifying the relationship between $\mathrm{El}$ and health. In this review, results of different investigations about $\mathrm{El}$ and its relationships with health and job performance in samples such as health (fitness center workers, surgeons and podiatrist) are presented. In addition, some key aspects are proposed to improve the El aiming at reducing stress, enhancing social and mental health and increasing the human resilience to different stressor agents (workload, disease or death of a relative, etc.).

KEYWORDS: Education, emotion, emotional intelligence, health, stress 


\section{Cómo citar el artículo:}

Simón Grima, J. (2020). Inteligencia emocional: retrato e importancia en profesionales de la salud (trabajadores del sector fitness, cirujanos y podólogos). Revista de Ciencias de la Comunicación e Información, 25(2), 23-40. doi: http://doi.org/10.35742/rcci.2020.25(2).23-40

\section{INTRODUCCIÓN}

El concepto de inteligencia ha sido definido por muchos autores en la literatura científica, y en humanos se ha estudiado en profundidad durante más de un siglo (Nisbett et ál. 2012). Sin embargo, científicos como William H. Calvin creen que jamás podrá lograrse definir de forma incontestable tal concepto por ser un vocablo abierto, al igual que la conciencia (Calvin, 1994). En la misma línea, otros autores también afirman que "la inteligencia" es muy compleja de definir y muy problemática para medir (Arsuaga y Martínez, 1999). No obstante, en términos generales, existe una definición que ha obtenido una amplia aceptación en la comunidad científica (Nisbett et ál., 2012). Ésta determina que la inteligencia es "la capacidad de razonar, planificar, resolver problemas, pensar de manera abstracta, comprender ideas complejas, aprender rápidamente y aprender de la experiencia. Por lo tanto, no se trata simplemente de aprendizaje de libros, una habilidad académica limitada 0 inteligencia para realizar exámenes. Más bien refleja una capacidad más amplia y profunda para comprender nuestro entorno: "comprender", "dar sentido" a las cosas o "averiguar qué hacer" (Gottfredson 1997, p. 13). Por ello, parece que la inteligencia general, tal como se define en humanos o animales no humanos, enfatiza la capacidad de razonamiento y la flexibilidad de comportamiento (Burkart et ál., 2017). También se cree que la inteligencia implica adquirir nuevos conocimientos a través de la interacción con el entorno (físico y social), así como resolver problemas novedosos y desconocidos que se presentan (Rumbaugh y Washburn, 2003).

Con respecto al origen de la inteligencia humana, no existe un consenso científico ni está demostrado que ésta se haya desarrollado tras un proceso lento y continuo ni que exista gracias a una causa sobrenatural. Ambas teorías creadas por Darwin y Wallace, respectivamente (Plotkin, 1997; Burkart et ál., 2017). Más allá de este hecho, la cognición ha sido definida por la American Phychological Association como "las habilidades de una persona para aprender, recordar, razonar, resolver problemas y emitir juicios sólidos, particularmente en contraste con la inteligencia emocional". Con respecto a esto, algunos autores sostienen que la inteligencia general es una abstracción estadística, no un rasgo cognitivo, y que la primera no garantiza inferencias sobre la naturaleza o evolución de la segunda (Burkart et ál., 2017).

La inteligencia se comenzó a evaluar a partir de la publicación de la escala métrica de la inteligencia de Binet-Simon (1905). Ese momento, considerado histórico en el ámbito de la psicología y psicometría, fue el punto de partida para la construcción, estandarización, adaptación y publicación de una gran cantidad de test y escalas de inteligencia para medir el nivel de ésta en las personas. La valoración de una capacidad global o coeficiente intelectual de inteligencia se pretende realizar a partir 
de la observación de un conjunto de funciones cognitivas supuestamente relacionadas con ella.

Tradicionalmente, siempre se ha considerado que la inteligencia cognitiva es una cualidad que se sitúa en el peldaño más alto del constructo humano, dejando a otras en escalones más bajos. No obstante, el empirismo y experiencia actual ha mostrado que ser inteligente a nivel cognitivo únicamente no garantiza el éxito académico, profesional y/o personal (Goleman, 1995; Jiménez y López-Zafra, 2009). Además, recientemente, el aspecto emocional ha cobrado más importancia en los ámbitos tanto laboral como educativo (Fernández-Berrocal y Aranda, 2008). Autores como Lazarus (1982) y Zajonc $(1980,1984)$ debatieron sobre ello y plantearon el tema de la disociación. Zajonc consideró el sistema emocional como férreo antagonista del sistema cognitivo. Lazarus, por su parte, lo integró en un contexto cognitivo, pero diferente del común.

\section{OBJETIVOS}

Uno de los objetivos del presente artículo es explicar y desarrollar el concepto de inteligencia emocional, instrumentos de evaluación y claves para su mejora y ejercitación, así como hacer una comparación entre ésta y la inteligencia cognitiva. Además, se propone como objetivo secundario repasar la literatura científica referente a la influencia de la inteligencia emocional sobre diferentes variables (relación con el usuario/paciente, empatía, estrés general, burnout, satisfacción laboral, etc.) en algunos de los profesionales de la salud que han sido menos estudiados en relación a ésta, como son profesionales del sector fitness, cirujanos y podólogos.

\section{Inteligencia emocional}

La inteligencia emocional (IE) es un concepto que fue popularizado por Goleman (1995) y que se define como la capacidad para percibir, asimilar, comprender y regular las emociones propias y las de los demás (Mayer y Salovey, 1997; Petrides y Furnham, 2003). A pesar de esta definición, la IE está conceptualizada por muchos autores y de formas muy diferentes. Encontramos una última definición de IE formulada recientemente por Barrientos (2019), quien la define como la capacidad de controlar y gestionar positivamente las emociones propias y ajenas, en un escenario cualquiera, donde se producen experiencias y cambios como parte del proceso de aprendizaje personal.

La importancia de la IE reside en que las emociones son un aspecto esencial de la naturaleza humana y estimulan la motivación hacia el comportamiento, de forma que ésta puede ser un potente indicador de éxito (en muchos aspectos de la vida) en el futuro (Goleman, 1995). A su vez, la IE es un importante predictor del funcionamiento social y personal de los seres humanos y, más concretamente, de la salud mental y social (Martins et ál., 2010). Cabe destacar que las emociones se centran en experiencias subjetivas que las personas representan, en parte, con miles de términos semánticos, y esta organización geométrica ha provocado un intenso debate (Cowen y Keltner, 2017). 
En la sociedad actual, las emociones desempeñan un papel cultural muy relevante (Zaccagnini, 2008). Durante la década de los años 70, el psicólogo Paul Eckman identificó seis emociones básicas que, en su opinión, tenían una experiencia universal en todas las culturas humanas. Las emociones que identificó fueron felicidad, tristeza, asco, miedo, sorpresa e ira. Más tarde amplió su lista de emociones básicas para incluir orgullo, lástima, vergüenza y excitación (Robinson et ál., 2013).

Por su parte, el psicólogo Robert Plutchik creó una "rueda de emociones" que funcionaba de manera similar a la rueda de colores. Las emociones podrían combinarse entre sí para formar diferentes sentimientos, al igual que se pueden mezclar los colores en una paleta. Las emociones más básicas se asemejarían a bloques de construcción, y las más complejas serían combinaciones de las más básicas. Por ejemplo, la alegría y la confianza (emociones básicas) se combinarían para crear el amor (emoción compleja) (Plutchik, 1991, 2001; Chafale y Pimpalkar, 2014).

Un reciente estudio realizado en 2017 por Cowen y Keltner sugiere que hay emociones mucho más básicas de lo que se creía con anterioridad. Estos investigadores identificaron 27 categorías diferentes de emociones. Sin embargo, en lugar de ser completamente distintos, los investigadores descubrieron que las personas experimentan estas emociones a lo largo de un gradiente.

Al igual que no todos tenemos el mismo coeficiente intelectual, tampoco somos similares en cuanto a IE, ya que, las personas difieren en la manera en que son capaces de identificar, expresar, usar y regular sus emociones y las de otros (Petrides et ál., 2016).

Se han propuesto diferentes modelos de IE, los cuales se han denominado "modelo de habilidad" (Mayer et ál., 2008) y "modelos mixtos o de rasgos", cuyo autor fundamental es Bar-on (1997) (figura 1). Existen otras propuestas que incluyen variables como personalidad y otras competencias (Goleman, 2001; Petrides y Furnham, 2006).

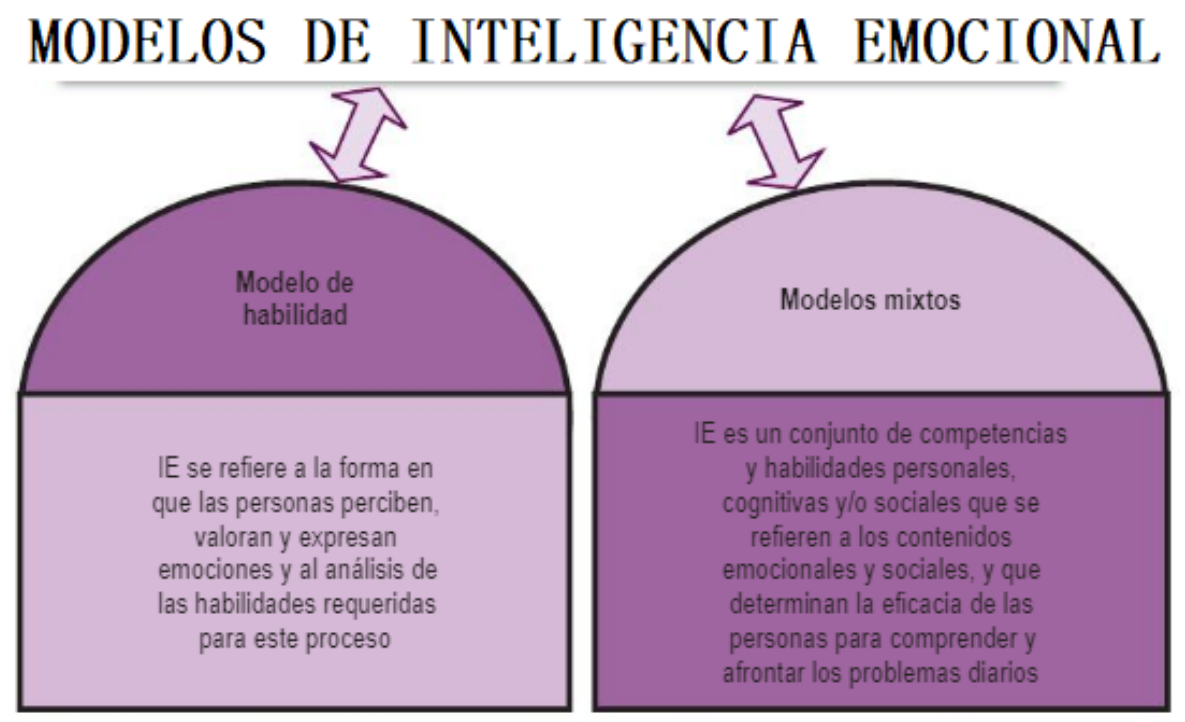

Figura 1. Modelos de inteligencia emocional.

Fuente: Extraído de Domínguez y Cameselle (2015). 


\subsection{Evaluación de la inteligencia emocional}

En opinión de Salovey y Mayer (1990), la IE debe de ser evaluada mediante ejercicios de resolución de problemas. Sin embargo, la puesta en marcha de la IE, como habilidad, es complicada debido a la subjetividad de las experiencias emocionales (Robinson y Clore, 2002). Una alternativa para su evaluación podría ser conceptualizarla como un rasgo medible a través de un auto-informe que plasmase la percepción subjetiva de las habilidades emocionales de un sujeto (Schutte et ál., 2001; Brackett y Geher, 2006). No obstante, este tipo de herramientas han sido criticadas debido a su gran dependencia de la auto-percepción (Matthews et ál., 2004). Sin embargo, ésta limitación es común a todas las escalas de auto-informe que se utilizan en el ámbito de la investigación y, por lo tanto, parece que no sería lógico descartar su uso para evaluar la IE (Davies et ál., 2010).

\subsection{Instrumentos para evaluar la inteligencia emocional}

Existen diferentes instrumentos para medir la inteligencia emocional (IE), los cuales constan de diversos ítems y un número de dimensiones determinadas. Salovey et ál. (1995) desarrollaron el Trait Meta-Mood Scale (TMMS-48), el cual consta de 48 ítems incluidos en 3 dimensiones. El TMMS-48 fue modificado, simplificado (de 48 ítems pasó a 24 ítems) y traducido al español por Extremera y Fernandez-Berrocal (2004). Más adelante, en el año 1997 y 1998, se crearon dos test más. Bar-On (1997) desarrolló el Emotional Quotient Inventory (EQ-i), con un gran número de ítems (133) englobados en 15 dimensiones, y validado en una muestra de 120 sujetos. Schutte et ál. (1998), por su parte, solamente incluyeron 33 ítems en el Schutte Self Report Inventory (SSRI). Éste fue validado en 346 personas. Uno de los instrumentos más completos para evaluar la IE fue el elaborado por Mayer et ál. (1999). Dicho test, el Emotional Intelligence Test (MSCEIT) consta de 141 ítems distribuidos en 4 dimensiones, y fue validado en una muestra de 5000 personas. También existen instrumentos validados para poblaciones específicas, como escolares. Ejemplo de ello es el Cuestionario de Inteligencia Emocional como rasgo para escolares (CIE), desarrollado por Mestre (2003), el cual consta de 56 ítems integrados en 4 dimensiones diferentes. Dicho test se validó en una amplia muestra de 1002 sujetos. En cuanto a número de ítems, el Trait Emotional Intelligence Questionnaire, elaborado por Petrides y Furnham, es el más completo, ya que consta de 144 ítems agrupados en 15 dimensiones. Dicho cuestionario se validó en una población de 282 sujetos.

En los últimos tiempos, el uso de las entrevistas como herramienta para evaluar la $\mathrm{IE}$, ha sido propuesta como una alternativa novedosa y válida. Sin embargo, algunos autores ponen en duda el rendimiento y la validez de éstas, ya que, en su opinión, la IE, la capacidad mental y la extraversión, son estudiadas en las entrevistas de forma aislada, sin obtener resultados destacables (Kluemper et ál., 2015). Otra perspectiva ofreció una revisión publicada en la revista Journal of Managerial Psychology, y cuyo objetivo era precisar y definir ese constructo en ocasiones difuso que es la IE, concluyó que ésta podría ser evaluada de forma más efectiva mediante "análisis de rendimiento" que con "pruebas clásicas con papel y lápiz". Además, éstos autores apoyan la idea de que la combinación de IE e inteligencia cognitiva o intelectual es 
un predictor de "éxito" más poderoso que cualquiera de las medidas aisladas (Dulewicz y Higgs, 2000).

\subsection{Estrategias para entrenar la inteligencia emocional}

Las claves propuestas para aprender a ser más inteligente a nivel emocional han sido las siguientes (Bobinski, 2010):

1) Las ideas de "bueno" o "malo" han de descartarse al pensar en estilos de personalidad. Las personas simplemente somos diferentes.

2) Las personas relacionan con frecuencia los términos "diferente" con "difícil". Sin embargo, lo diferente es difícil solamente porque las personas no han aprendido a trabajar con efectividad esas diferencias.

3) Todas las fortalezas tienen una debilidad asociada y todas las debilidades tienen una fortaleza asociada. Debemos de centrarnos en qué extremo queremos trabajar.

4) Debemos de focalizarnos en las fortalezas en vez de en las debilidades. Todos los estilos de personalidad suman fortaleza a un equipo.

5) Hay que esforzarse constantemente por encontrar las fortalezas en cada estilo de personalidad.

6) No podemos ser efectivos si esperamos que las personas no salgan de su zona de confort.

7) Debemos de preguntar a los demás lo que significa un término determinado (ganar, perder, mejorar, etc.). No podemos asumir que conocemos la respuesta.

8) Anteponer nuestros objetivos a los del resto creará divisiones y debilitará la habilidad para maximizar resultados

9) Efectividad (hacer lo correcto) y eficiencia (hacerlo rápido) rara vez van unidas. Los mejores resultados se obtienen cuando nos tomamos el tiempo necesario para hacer las cosas correctamente a través de un trabajo multidisciplinar.

10) Comprender estas claves no es lo mismo que llevarlas a cabo. En ocasiones la distancia más larga es la que se encuentra entre cabeza y corazón.

\subsection{Importancia de la inteligencia emocional en los trabajadores de la salud}

La IE ha cobrado gran relevancia debido a que la gran cantidad de estudios realizados muestran que ésta beneficia a múltiples áreas del funcionamiento humano (Joseph y Newman, 2010; Bracket et ál., 2011). Sin embargo, sus efectos no son directos, sino que se producen debido a que las personas con una alta IE mantienen relaciones interpersonales positivas y satisfactorias (Fernández-Berrocal y Aranda, 2008). Por lo tanto, adquirir esta serie de habilidades podría ayudar a resolver algunos de los problemas existentes en la actualidad en distintos contextos, concretamente el sanitario y laboral (Sabucedo y Morales, 2015).

Una de las necesidades más importantes del ser humano es tener una buena salud, lo cual es indicador de la calidad de vida y un requisito fundamental para ser feliz (Hitam y Borhan, 2012). Por ello, gobiernos y diferentes entidades se implican fuertemente en trabajar para mejorar la salud pública y, por lo tanto, la salud general 
de la población (Asiamah, 2017). Dentro de las medidas que se están tomando, mejorar la atención médica y el rendimiento laboral de los sanitarios es una prioridad. Por ello, gran parte del gasto en promoción de la salud pública de todo el mundo se ha destinado para este fin (Diab y Ajlouni, 2015).

Si se plantea mejorar la calidad de vida de la población general y el rendimiento laboral en sanitarios, por ejemplo, el control de los niveles de estrés debería de ser crucial para lograr este objetivo ya que, vivimos en una sociedad muy estresada. Según la American Psychological Association (APA), la mayoría de los ciudadanos estadounidenses reportan niveles de estrés de moderados a altos (Am. Psychol. Assoc. 2012). Además, la OMS proclamó el siglo XX como "el siglo de las tensiones colectivas e individuales en proporciones epidémicas, no vistas hasta ahora en la historia de la raza humana". Los sistema y procesos fisiológicos involucrados en el estrés han evolucionado para ayudarnos a afrontar o escapar de situaciones que amenazaban nuestras vidas (luchar o huir) (Lovallo y Thomas, 2000). Sin embargo, en la actualidad, la mayoría de los factores estresantes a los que nos enfrentamos a diario son psicológicos (relacionados con nuestro puesto de trabajo, por ejemplo) en lugar de físicos (Tomiyama, 2018). Esto es de vital importancia ya que, durante el siglo pasado, el estrés se convirtió en un factor crítico para mantener la salud y la causa de las enfermedades psicosomáticas (Burgard et ál., 2019).

Debido a que los niveles de estrés en el personal dedicado a la salud son elevados, sobre todo en algunas áreas y especialidades (Aguado et ál., 2013), la forma en que un profesional de esta rama afronte ese agente estresor le hará más fuerte o más débil. Ésta persona se distinguirá de otra por su capacidad para percibir, comprender y regular sus emociones y la de los demás cuando se expone a un estrés general o generado por una circunstancia en particular. Cuanta más facilidad tenga una persona para identificar una emoción durante una situación de estrés, menos tiempo invertirá en atender a sus reacciones emocionales y menos recursos cognitivos utilizarán. Esto es positivo, ya que podrá evaluar más alternativas de acción y mantener pensamientos en otras tareas o bien llevar a cabo estrategias de afrontamiento activas (Gohm y Clore, 2002; Leung et ál., 2008). Esta capacidad, inherente a la IE, también tiene mucho que ver con la psicología positiva (o negativa), una nueva forma de analizar los procesos básicos frente a los traumas, trastornos y patologías (Seligman, 2002). Además, varios son los estudios que han confirmado un efecto positivo significativo de la IE en el desempeño laboral (Olakitan, 2014; Özer et ál., 2016).

\subsection{La inteligencia emocional en trabajadores del sector fitness}

La Organización Mundial de la Salud (OMS) reconoció como enfermedad el "burnout" o "síndrome del trabajador quemado". En relación a ello, el psicólogo Herbert Freudenberger definió esta afección como "un síndrome de cansancio emocional, despersonalización, y una menor realización personal que se da en aquellos individuos que trabajan en contacto con clientes y usuarios" (Freudenberger 1986,1989). Esta definición es importante porque hace hincapié en aquellos profesionales que tienen un trato diario con personas, por ejemplo, entrenadores personales, coordinadores y/o monitores de centros deportivos, en gran parte representados por los licenciados/graduados en Ciencias de la Actividad Física y el 
Deporte. Estudios realizados con profesionales de la salud que trabajan directamente con personas han mostrado que, a mayor nivel de inteligencia emocional (IE), menor burnout y mayor satisfacción laboral (Weng et ál., 2011; Kim et ál., 2016). Bajo nuestro conocimiento, existen en la literatura científica pocos estudios que analicen la importancia de la inteligencia emocional en trabajadores del sector fitness en concreto. Uno de estos estudios, llevado a cabo por Ong y Yap (2017) analizó una muestra de 217 empleados de centros deportivos, los cuales completaron cuestionarios para medir su IE y agotamiento profesional, entre otros. Los resultados mostraron que la aptitud en cuanto a IE y la forma en que los trabajadores gestionan sus emociones podrían afectar al bienestar de éstos. Además, se observó que la IE desempeña un papel importante en la selección de estrategias (beneficiosas o perjudiciales) para la salud entre los empleados de los centros fitness.

También se ha observado que un deportista que perciba que su entrenador gestiona bien sus emociones y sabe tratar a todos por igual, ayudando a la mejora personal de cada uno de ellos, mejorará su motivación intrínseca (González-Vélez, 2010). Otros estudios realizados en amplias muestras de profesionales de la salud (1163 sujetos), han concluido que la IE predice significativamente el rendimiento laboral en este tipo de población (Asiamah, 2017). Además, estudios realizados en entrenadores de diferentes disciplinas $(n=99)$ deportivas mostraron que la IE se relaciona con la eficacia en el entrenamiento y da pistas de hacia dónde se puede dirigir el trabajo aplicado con los entrenadores (Thelwell et ál., 2008). No obstante, y a pesar de que sean trabajos muy relacionados, en muchas ocasiones un profesional del sector fitness busca la mejora de la salud del usuario y no el rendimiento únicamente.

A pesar de que la experiencia y algunos estudios científicos nos dicen que la IE podría ser una capacidad de vital importancia para el desempeño laboral y la mejora en la relación con los usuarios en profesionales del sector fitness, todavía no contamos con suficientes investigaciones específicas realizadas en esta población que puedan resolver estas dudas. Se hace necesaria más investigación que estudie a los profesionales del sector fitness (entrenadores personales, monitores deportivos, coordinadores deportivos, etc.) y los beneficios que la IE puede ofrecer a este sector.

\subsection{Cirujanos e inteligencia emocional}

Desde hace un tiempo, el mundo de la medicina ha comenzado a entender, valorar y reconocer los posibles beneficios de la IE tanto para los pacientes como para los trabajadores sanitarios (Jensen et ál. 2008). Dentro del amplio grupo de profesionales que constituyen el campo de la salud, se ha demostrado que un tercio de los cirujanos americanos sufrían grados elevados de burnout (Campbell et ál., 2001). Parece ser que la edad, la antigüedad laboral y el número de días de vacaciones fueron las variables demográficas asociadas inversamente a éste síndrome. Otros estudios han mostrado entre los cirujanos un grado moderado de burnout con una satisfacción laboral media, justificada por el carácter vocacional de éstos (Jiménez et ál., 2002). Además, en cirujanos de trasplante, se observaron grados todavía más altos de burnout que en el estudio de Campbell et ál. (2001). Por 
ello, desde hace años se han propuesto diferentes estrategias de afrontamiento y prevención que podrían ser muy útiles para mejorar el manejo de determinadas demandas específicas externas y/o internas, así como la gestión de recursos en respuesta al estrés (Lazarus, 1997; Ceslowitz, 1989). Como se comentó anteriormente, la IE se refiere al conjunto de competencias y habilidades que determinaran la eficacia de las personas para gestionar, comprender y afrontar los problemas diarios. Por ello, es importante revisar la literatura científica en busca de resultados que arrojen luz sobre el papel de la IE en cirujanos.

Con respecto a esto, en el estudio llevado a cabo por Weng et ál. (2011) en el que se encuestó a 50 cirujanos y a sus 549 pacientes, se observó que la IE de los cirujanos tuvo un efecto positivo en las relaciones entre éstos y los pacientes. En relación a esto, también se ha asociado un mayor nivel de IE en una muestra de 126 cirujanos con un menor agotamiento profesional en éstos (Benson et ál., 2007). Además, dentro de la escala general de la IE, parecen existir diferencias en las subescalas entre cirujanos y otros profesionales de la salud. Por ejemplo, un estudio publicado por la Universidad de Cambridge en el cual se comparó la IE entre cirujanos y psiquiatras $(n=148)$, concluyó que a pesar de que la puntuación total promedio de ésta en ambas poblaciones no difirió, los cirujanos mostraron puntuaciones más altas en las sub-escalas de autoestima, tolerancia al estrés y optimismo en comparación con los psiquiatras (Stanton et ál., 2011). Resultados como éstos podrían indicar la necesidad de entrenar y trabajar diferentes habilidades concretas en función del profesional de la salud implicado. Con respecto a esto, una reciente revisión de la literatura científica (39 artículos y 5 libros de texto) cuyo objetivo fue abordar las implicaciones de la IE en cirujanos, concluyó que los cirujanos deberían de conocer sus propias emociones y las de los demás para poder desempeñar de forma óptima su trabajo. En esta revisión también se concluyó que la IE está asociada positivamente con el liderazgo en cirujanos, habilidades no técnicas, disminución del estrés, menor agotamiento laboral y una mayor satisfacción laboral (Sharp et ál., 2020).

\subsection{Inteligencia emocional en el ámbito de la podología}

La mayor parte de la literatura científica que estudia el agotamiento laboral en profesionales de la salud se ha centrado en enfermería, fisioterapia (Janssen et ál., 1999; Evans y Allen, 2002) y terapia ocupacional (Scutter y Goold, 1995; Schuster et ál., 1984). Sin embargo, esta variable ha sido poco estudiada en podología. Debido a que se ha observado que los niveles de agotamiento en podólogos exceden de los valores normales establecidos para todos los profesionales de la salud (Mandy y Mandy, 2000), es importante valorar el papel de la inteligencia emocional en este grupo de trabajadores.

Dos motivos que justificarían estos valores altos de agotamiento profesional en comparación con otras profesiones (fisioterapeutas, por ejemplo) serían el aislamiento en el lugar de trabajo y la falta de respeto de otras disciplinas profesionales hacia los podólogos (Mandy y Mandy, 2000). También se ha sugerido que el agotamiento profesional se desarrolla con el tiempo a medida que se pierde el idealismo (Mandy y Tinley, 2002). Éstas variables podrían ser diferentes en función del país ya que, en Australia, por ejemplo, el $75 \%$ de los podólogos trabajan en 
centros privados (Op.cit), mientras que en España casi la totalidad de éstos lo hacen en este tipo de centros, siendo el porcentaje de podólogos que trabajan en centros públicos muy pequeños. Esto podría suponer que éstos profesionales sintiesen que no son apoyados por el resto de "colegas" sanitarios (Cherniss, 1995). Otros estudios afirman que trabajar más horas y las altas demandas emocionales que supone trabajar con pacientes podría incentivar dicho agotamiento laboral (Schaufeli y Enzmann, 1998). Además, en podólogos estudiados tanto en el Reino Unido como en Australia, se han observado también altos niveles de agotamiento emocional y bajos niveles de realización personal, ambos resultados sinónimos del síndrome de burnout (Mandy y Tinley, 2004). También se ha descrito agotamiento en podólogos como resultado de trabajar principalmente con el grupo de pacientes que generalmente se presentan a los servicios de podología (Op.cit; Potter y Borthwick, 2009). Debido a esto y a que la IE es vital para ayudar a superar este síndrome, así como para que el podólogo mejore el bienestar emocional del paciente y su propio plan de acción terapéutico (Ivcevic et ál., 2007), se hace necesario repasar que ha aportado la literatura científica con respecto a la IE y la podología.

Existe poca literatura científica que se haya centrado en cómo afectan los niveles de IE en podólogos a su rendimiento, salud e interacción con los pacientes. Uno de estos estudios ha concluido que en estudiantes de podología existe una fuerte correlación entre la empatía, la personalidad y la IE de éstos (Bertram et ál., 2016). Si bien esta muestra poblacional todavía no eran podólogos de profesión, son sujetos que lo iban a ser en poco tiempo. También se ha afirmado que debido a que el podólogo puede desarrollar una relación profesional a largo plazo con el paciente (afecciones crónicas que afectan a la marcha, quiropodias rutinarias, etc.), pudiese ser que la disminución de la salud o la muerte de éstos afectasen al bienestar mental y emocional del profesional, debido a que no están preparados para lidiar con esta situación. En relación con este hecho, un estudio que analizó a 15 podólogos del sector privado, sugiere que existe un profundo impacto en el podólogo cuando se produce una muerte de un paciente que ha sido tratado durante bastante tiempo (Robson y Williams, 2017). Por ello, se propone implementar estrategias para afrontar a nivel emocional estas situaciones (Carton y Hupcey, 2014). Los podólogos incluidos en éste estudio también afirmaron no tener capacitación formal sobre cómo lidiar con el impacto psicológico asociado a la muerte de un paciente a largo plazo. Unas de las intervenciones positivas se deberían de focalizar en desarrollar la resiliencia en estos profesionales, la cual ha sido asociada a la inteligencia emocional (Edward y Warelow, 2005; Maulding et ál., 2012; Schneider et ál. 2013).

\section{CONCLUSIONES}

En los últimos años, la investigación en psicología se ha centrado en el estudio de la IE, la cual comprende un conjunto de habilidades y competencias orientadas a comprender, analizar y dar respuestas óptimas ante situaciones diarias que impliquen un mayor o menor nivel emocional. Esta inteligencia, hasta hace unas décadas desconocida, ha demostrado ser un factor predictivo del "éxito" a nivel personal, laboral y académico. Hasta que este hecho se ha dado, la inteligencia cognitiva (en el contexto de los seres humanos) acaparaba toda (o la mayor parte) de la importancia y se situaba en el escalón más alto. Sin embargo, eso ha cambiado gracias a los nuevos avances científicos. Si bien es cierto que cuando una 
persona tiene niveles altos de ambos tipos de inteligencia puede tener más probabilidades de "éxito", ambas parecen ser independientes y tener un nivel alto en una de ellas no implicaría forzosamente tener un nivel alto en la otra. Existen diversos instrumentos para evaluar la IE. Sin embargo, también hay controversia sobre cuáles son los más efectivos o miden mejor este conjunto de habilidades. Los métodos más utilizados para evaluarla son los cuestionarios auto-informados, aunque también se han propuesto las entrevistas o las "pruebas de rendimiento concretas".

Existen diferentes estrategias y claves para mejorar la inteligencia emocional, y estas pasan por trabajar con constancia cada una de las habilidades que ésta comprende, enfocándonos en las debilidades (para mejorarlas), pero también en las fortalezas (para reforzarlas). Tener mayor niveles de IE nos hará empatizar más fuertemente con los demás, mejorar nuestras relaciones sociales, disminuir nuestros niveles de estrés, reducir el agotamiento profesional (incluidos profesionales de la salud sometidos a altos niveles de estrés y en contacto directo con usuarios/pacientes), gestionar mejor diferentes agentes estresores e implementar estrategias de afrontamiento más efectivas contra estos, gestionar nuestras emociones y las de los demás, aumentar nuestra resiliencia, así como mejorar nuestra calidad de vida tanto física como mental.

\section{REFERENCIAS}

Aguado Martín, J. I., Bátiz Cano, A., y Quintana Pérez, S. (2013). El estrés en personal sanitario hospitalario: estado actual. Medicina y Seguridad del trabajo, 59(231), 259-275.

American Psychological Association. (2012). Guidelines for psychological practice with lesbian, gay, and bisexual clients. The American Psychologist, 67(1), 10.

Arsuaga, J. L., Gracia, A., Lorenzo, C., Martínez, I., y Pérez, P. J. (1999). Resto craneal humano de Galería/Cueva de los Zarpazos (Sierra de Atapuerca, Burgos). In Atapuerca: Ocupaciones humanas y paleoecología del yacimiento de Galería, 7, (233-235). Junta de Castilla y León.

Asiamah, N. (2017), "The nexus between health workers' emotional intelligence and job performance: Controlling for gender, education, tenure and in-service training", Journal of Global Responsibility, Vol. 8 No. 1, pp. 10-33. Asiamah, N. (2017). The nexus between health workers' emotional intelligence and job performance. Journal of Global Responsibility.

Bar-On, R. (1997). BarOn emotional quotient inventory. Multi-health systems.

Barrientos-Báez, A., Barquero-Cabrero, M., y Rodríguez-Terceño, J. (2019). Educación emocional como contenido transversal para una nueva política educativa: el caso del Grado de Turismo. Utopía y Praxis Latinoamericana, 24(4), 147-165. ISSN 1315-5216. 
Benson S. Truskett P.G. Findlay B. The relationship between burnout and emotional intelligence in Australian surgeons and surgical trainees. ANZ J Surg 2007; 77 ((suppl 1)).

Berrocal, P. F. y Aranda, D. R. (2008). La educación de la inteligencia emocional desde el modelo de Mayer y Salovey. In Educación emocional y convivencia en el aula, 163-178. Subdirección General de Información y Publicaciones

Bertram, K., Randazzo, J., Alabi, N., Levenson, J., Doucette, J. T. y Barbosa, P. (2016). Strong correlations between empathy, emotional intelligence, and personality traits among podiatric medical students: A cross-sectional study. Education for Health, 29(3), 186.

Binet, A., y Simon, T. (1905). Upon the necessity of establishing a scientific diagnosis of inferior states of intelligence (ES Kite, trans.). A. Binet y T. Simon, The development of intelligence in children (The Binet-Simon Scale), 37-90.

Bobinski, D. (2010). Ten secrets of Emotional Intelligence. http://www.managementissues.com/opinio/5900/ten-secrets-of-emotionalinteligence

Brackett, M. A., y Geher, G. (2006). Measuring emotional intelligence: Paradigmatic diversity and common ground. Emotional intelligence in everyday life, 2, 27-50.

Brackett, M. A., Palomera, R., Mojsa-Kaja, J., Reyes, M. R., y Salovey, P. (2010).Emotion-regulation ability, burnout, and job satisfaction among British secondary-school teachers. Psychology in the Schools, 47(4), 406-417.

Burgard, T., Bosnjak, M., y Kasten, N. (2019). Moderators of panel conditioning effects. A meta-analysis. Presentation given at the 21st General Online Research Conference, TH Köln, Cologne.

Burkart, J. M., Schubiger, M. N., y Van Schaik, C. P. (2017). The evolution of general intelligence. Behavioral and Brain Sciences, 40.

Calvin, W. H. (1994). The emergence of intelligence. Scientific American, 271(4), 100-107.

Campbell Jr, D. A., Sonnad, S. S., Eckhauser, F. E., Campbell, K. K., y Greenfield, L. J. (2001). Burnout among American surgeons. Surgery, 130(4), 696-705.

Carton, E. R., y Hupcey, J. E. (2014). The forgotten mourners: addressing health care provider grief-a systematic review. Journal of Hospice y Palliative Nursing, 16(5), 291-303.

Ceslowitz, S. B. (1989). Burnout and coping strategies among hospital staff nurses. Journal of advanced nursing, 14(7), 553-557. 
Chafale, D., y Pimpalkar, A. (2014). Review on developing corpora for sentiment analysis using plutchik's wheel of emotions with fuzzy logic. International Journal of Computer Sciences and Engineering (IJCSE), 2(10), 14-18.

Cherniss, C. (1995) Beyond Burnout: Helping teachers, nurses, therapists and lawyers recover from stress and disillusionment. Routledge.

Cowen, A. S., y Keltner, D. (2017). Self-report captures 27 distinct categories of emotion bridged by continuous gradients. Proceedings of the National Academy of Sciences, 114(38), E7900-E7909.

Davies, K. A., Lane, A. M., Devonport, T. J., y Scott, J. A. (2010). Validity and reliability of a brief emotional intelligence scale (BEIS-10). Journal of Individual Differences, 31(4), 198-208

Diab, S. M., y Ajlouni, M. T. (2015). The influence of training on employee's performance, organizational commitment, and quality of medical services at Jordanian private hospitals. International Journal of Business and Management, $10(2), 117$.

Domínguez, M., y Cameselle, S. (2015). Psicología social: la ciencia de la persona y la sociedad. In Psicología social, 1-19. Editorial Médica Panamericana.

Dulewicz, V. y Higgs, M. (2000). Emotional intelligence. A review and evaluation study. Journal of Managerial Psychology, 15(4):341-372

Edward, K. L. y Warelow, P. (2005). Resilience: When coping is emotionally intelligent. Journal of the American Psychiatric Nurses Association, 11(2), 101102.

Evans, D., y Allen, H. (2002). Emotional intelligence: its role in training. Nursing times, 98(27), 41-42.

Extremera, N. y Fernández-Berrocal, P. (2005). Perceived emotional intelligence and life satisfaction: Predictive and incremental validity using the Trait Meta-Mood Scale. Personality and Individual Differences, 39(5), 937-948.

Fernández-Berrocal, P. y Aranda, D. R. (2017). La inteligencia emocional en la educación. Electronic Journal of Research in Education Psychology, 6(15), 421436.

Freudenberger, H. J. (1986). The issues of staff burnout in therapeutic communities. Journal of psychoactive drugs, 18(3), 247-251.

Freudenberger, H. J. (1989). Burnout: Past, present, and future concerns. Loss, Grief y Care, 3(1-2), 1-10.

Furnham, A. y Petrides, K. V. (2003). Trait emotional intelligence and happiness. Social Behavior and Personality: an international journal, 31(8), 815-823. 
Gohm, C. L. y Clore, G. L. (2002). Four latent traits of emotional experience and their involvement in well-being, coping, and attributional style. Cognition \& Emotion, 16(4), 495-518.

Goleman, D. (1995). Inteligencia Emocional. Editorial Bantam Books.

Goleman, D. (2001). Emotional intelligence: Issues in paradigm building. The emotionally intelligent workplace, 13, 26.

González-Vélez, J. L. (2010). Inteligencia emocional y motivación en el deporte. (Tesis Doctoral). Universidad de Las Palmas de Gran Canaria.

Gottfredson, L. S. (1997). Why g matters: The complexity of everyday life. Intelligence, 24(1), 79-132.

Hitam, M. B. y Borhan, H. B. (2012). FDI, growth and the environment: impact on quality of life in Malaysia. Procedia-Social and Behavioral Sciences, 50, 333-342.

Ivcevic, Z., Brackett, M. A. y Mayer, J. D. (2007). Emotional intelligence and emotional creativity. Journal of personality, 75(2), 199-236.

Janssen, P. P., De Jonge, J. y Bakker, A. B. (1999). Specific determinants of intrinsic work motivation, burnout and turnover intentions: a study among nurses. Journal of advanced nursing, 29(6), 1360-1369.

Jensen, A. R., Wright, A. S., Lance, A. R., O’Brien, K. C., Pratt, C. D., Anastakis, D. J., ... y Horvath, K. D. (2008). The emotional intelligence of surgical residents: a descriptive study. The American Journal of Surgery, 195(1), 5-10.

Jiménez, C., Morales, J. L. y Martínez C. (2002). Estudio del síndrome de burnout en cirujanos pediatras españoles. Cir Pediatr, 15, 73-8.

Jiménez Morales, M. I. y López-Zafra, E. (2009). Emotional intelligence and school performance: An updated overview. Revista Latinoamericana de Psicología, 41(1), 69-79.

Joseph, D. L., y Newman, D. A. (2010). Emotional intelligence: an integrative metaanalysis and cascading model. Journal of applied psychology, 95(1), 54.

Kim, J. H., Yoon, Y. J., y Jung, I. K. (2016). The mediating effects of stress coping strategy on the relationship between academic burnout and school adjustment in Korean adolescents. Journal of Korean Home Economics Education Association, 28(1), 71-85.

Kluemper, D. H., McLarty, B. D., Bishop, T. R., y Sen, A. (2015). Interviewee selection test and evaluator assessments of general mental ability, emotional intelligence and extraversion: Relationships with structured behavioral and situational interview performance. Journal of Business and Psychology, 30(3), 543-563. 
Lazarus, R. S. (1982). Thoughts on the relations between emotion and cognition. American psychologist, 37(9), 1019.

Lazarus, R. S. (1984). On the primacy of cognition. American Psychologist, 39(2), 124-129

Leung, M. Y., Chan, Y. S. y Olomolaiye, P. (2008). Impact of stress on the performance of construction project managers. Journal of Construction Engineering and Management, 134(8), 644-652.

Lovallo, W. R. y Thomas, T. L. (2000). Stress hormones in psychophysiological research: Emotional, behavioral, and cognitive implications.

Mandy, A. y Mandy, P. (2000). Burnout and work stress in dentistry and podiatry. $J$ Occup Health, 52, 25.

Mandy, A. y Tinley, P. (2002). Burnout and occupational stress in Australian podiatrists. Australasian Journal of Podiatric Medicine, 36(4), 101-108.

Mandy, A. y Tinley, P. (2004). Burnout and occupational stress: comparison between United Kingdom and Australian podiatrists. Journal of the American Podiatric Medical Association, 94(3), 282-291.

Martins, A., Ramalho, N. y Morin, E. (2010). A comprehensive meta-analysis of the relationship between emotional intelligence and health. Personality and Individual Differences, 49, 554-564

Maulding, W. S., Peters, G. B., Roberts, J., Leonard, E., y Sparkman, L. (2012). Emotional intelligence and resilience as predictors of leadership in school administrators. Journal of Leadership Studies, 5(4), 20-29.

Mayer, J. D. y Salovey, P. (1997). Emotional development and emotional intelligence: Implications for educators. What is emotional intelligence, 5.

Mayer, J. D., Salovey, P. y Caruso, D. R. (2008). Emotional intelligence: New ability or eclectic traits?. American psychologist, 63(6), 503.

Mayer, J.D., Caruso, D. y Salovey, P. (1999). Emotional intelligence meets traditional standards for an intelligence. Intelligence, 27, 267-298.

Mestre, J. M. (2003). Validación empírica de una prueba para medir la inteligencia emocional en una muestra de estudiantes de la bahía de Cádiz. Tesis Doctoral, Universidad de Cádiz.

Nisbett, R. E., Aronson, J., Blair, C., Dickens, W., Flynn, J., Halpern, D. F. y Turkheimer, E. (2012). Intelligence: new findings and theoretical developments. American psychologist, 67(2), 130. 
Olakitan, O. O. (2014). Emotional intelligence and perceived leadership behaviour effectiveness in organizations. International Journal of Humanities and Social Science, 4(2), 262-269.

Ong, D. L. T. y Yap, W. X. (2017). The impact of fitness center servicescape on individual behavior: The mediating role of emotional response. Journal of Global Sport Management, 2(2), 128-142.

Özer, E., Hamarta, E. y Deniz, M. E. (2016). Emotional intelligence, core-self evaluation, and life satisfaction. Psychology, 7(2), 145-153.

Petrides, K. V. y Furnham, A. (2006). The Role of Trait Emotional Intelligence in a Gender-Specific Model of Organizational Variables 1. Journal of Applied Social Psychology, 36(2), 552-569.

Petrides, K. V., Mikolajczak, M., Mavroveli, S., Sanchez-Ruiz, M. J., Furnham, A. y Pérez-González, J. C. (2016). Developments in trait emotional intelligence research. Emotion Review, 8(4), 335-341.

Plotkin, H. C. (1997). Darwin machines and the nature of knowledge. Harvard University Press.

Plutchik, R. (1991). The emotions. University Press of America.

Plutchik, R. (2001). The nature of emotions: Human emotions have deep evolutionary roots, a fact that may explain their complexity and provide tools for clinical practice. American scientist, 89(4), 344-350.

Potter, M. y Borthwick, A. (2009). Journal of foot and ankle research--JFAR: the official journal of the Society of Chiropodists and Podiatrists and the Australasian Podiatry Council. Podiatry Now, 12(5), 2-2.

Robinson, A., Kosmerly, S., Mansfield-Green, S. y Lafrance, G. (2013). Disordered eating behaviours in an undergraduate sample: Associations among gender, body mass index, and difficulties in emotion regulation. Canadian Journal of Behavioural Science, 46(3)

Robinson, M. D. y Clore, G. L. (2002). Belief and feeling: evidence for an accessibility model of emotional self-report. Psychological bulletin, 128 (6), 934.

Robson, K. y Williams, C. M. (2017). Dealing with the death of a long term patient; what is the impact and how do podiatrists cope?. Journal of foot and ankle research, 10(1), 36.

Rumbaugh, D. M. y Washburn, D. A. (2003). Intelligence of apes and other rational beings. New Haven: Yale University Press.

Salovey, P. y Mayer, J. D. (1990). Emotional intelligence. Imagination, cognition and personality, 9 (3), 185-211. 
Salovey, P., Mayer, J. D., Goldman, S. L., Turvey, C. y Palfai, T. P. (1995). Emotional attention, clarity, and repair: Exploring emotional intelligence using the Trait MetaMood Scale. In J. W. Pennebaker (Ed.), Emotion, disclosure, \& health (p. 125154). American Psychological Association.

Schaufeli, W. y Enzmann, D. (1998). The burnout companion to study and practice: A critical analysis. CRC press.

Schneider, T. R., Lyons, J. B. y Khazon, S. (2013). Emotional intelligence and resilience. Personality and Individual Differences, 55(8), 909-914.

Schutte, N. S., Malouff, J. M., Bobik, C., Coston, T. D., Greeson, C., Jedlicka, C., ... y Wendorf, G. (2001). Emotional intelligence and interpersonal relations. The Journal of social psychology, 141(4), 523-536.

Schutte, N. S., Malouff, J. M., Hall, L. E., Haggerty, D. J., Cooper, J. T., Golden, Ch. J. y Dornhein, L. (1998). Development and validation of a measure of emotional intelligence. Personality and Individual Differences, 25(2), 167-177.

Scutter, S. y Goold, M. (1995). Burnout in recently qualified physiotherapists in South Australia. Australian Journal of Physiotherapy, 41(2), 115-118.

Seligman, M. E. (2002). Positive psychology, positive prevention, and positive therapy. Handbook of positive psychology, 2, 3-12.

Sharp, G., Bourke, L. y Rickard, M. J. (2020). Review of emotional intelligence in health care: an introduction to emotional intelligence for surgeons. ANZ Journal of Surgery, 1-8

Stanton, C., Sethi, F. N., Dale, O., Phelan, M., Laban, J. T., y Eliahoo, J. (2011). Comparison of emotional intelligence between psychiatrists and surgeons. The Psychiatrist, 35(4), 124-129.

Thelwell, R. C., Lane, A. M., Weston, N. J., y Greenlees, I. A. (2008). Examining relationships between emotional intelligence and coaching efficacy. International Journal of Sport and Exercise Psychology, 6(2), 224-235.

Tomiyama, A. J. (2019). Stress and obesity. Annual review of psychology, 70, 703718.

Trait, E. I. (2004). Emotional intelligence. Psychologist, $17(10), 574$.

Weng, H. C., Hung, C. M., Liu, Y. T., Cheng, Y. J., Yen, C. Y., Chang, C. C. y Huang, C. K. (2011). Associations between emotional intelligence y doctor burnout, job satisfaction y patient satisfaction. Medical Education, 45, 835-842.

Zaccagnini, J. L. (2008). La comprensión de la emoción: una perspectiva psicológica. En M. S. Jiménez (coord.). Educación emocional y convivencia en el aula. Madrid. Ministerio de Educación, Política social y Deporte. 
Zajonc, R. B. (1980). Feeling and thinking: Preferences need no inferences. American psychologist, 35(2), 151.

\section{AUTOR:}

\section{Javier Simón Grima}

Obtuve la titulación de grado superior en Técnico superior en Animación de Actividades Físicas y Deportivas con matrícula de honor. Me gradué en Ciencias de la Actividad Física y el Deporte en la Universidad de Zaragoza (Facultad de Huesca). Posteriormente, me marché a Valencia para realizar el máster oficial en Fisiología (Universitat de València. Facultad de Medicina), obteniendo matrículas de honor en diversas asignaturas. Tras terminar dicho máster, me matriculé en el programa de doctorado en Ciencias de la Salud y el Deporte de la Universidad de Zaragoza, el cual finalicé con una calificación de cum laude. A día de hoy cuento con 6 publicaciones científicas en revistas de alto impacto y un capítulo de libro.

javisimongrima@gmail.com

Research Gate: https://www.researchgate.net/profile/Javier Simon-Grima 